\title{
Influence of Occupational and Environmental Exposure to Low Concentrations of Polychlorobiphenyls and a Smoking Habit on the Urinary Excretion of Corticosteroid Hormones
}

\author{
Maria Nicolà D'Errico ${ }^{1}$, Piero Lovreglio ${ }^{1, *}$, Ignazio Drago ${ }^{1}$, Pietro Apostoli ${ }^{2}$ and \\ Leonardo Soleo ${ }^{1}$ \\ 1 Section of Occupational Medicine "Enrico Carlo Vigliani", Interdisciplinary Department of Medicine, \\ University of Bari, Piazza G. Cesare 11, Bari 70124, Italy; marianicola.derrico@uniba.it (M.N.D.); \\ ignazio.drago@gmail.com (I.D.); leonardo.soleo@uniba.it (L.S.) \\ 2 Section of Occupational Medicine and Industrial Hygiene, Department of Experimental and Applied \\ Medicine, University of Brescia, Piazzale Spedali Civili 1, Brescia 25123, Italy; pietro.apostoli@unibs.it \\ * Correspondence: piero.lovreglio@uniba.it; Tel.: +39-080-5478-218
}

Academic Editor: Huixiao Hong

Received: 18 January 2016 ; Accepted: 15 March 2016 ; Published: 25 March 2016

\begin{abstract}
The effects of occupational exposure to low concentrations of polychlorobiphenyls (PCBs) on the urinary excretion of corticosteroid hormones were evaluated, taking into account the influence of cigarette smoking. The study included 26 males working as electrical maintenance staff in a steel factory, previously exposed to a mixture of PCBs (exposed workers), and 30 male workers with no occupational exposure to PCBs (controls). Serum PCBs (33 congeners), urinary 17-hydroxycorticosteroids, 17-ketosteroids (KS) and pregnanes, and their respective glucuronidated and sulfonated compounds, were determined for each subject. PCBs were significantly higher in the exposed workers than controls, and were correlated with age. Both the urinary concentrations of the total 17-KS and pregnanes, and those of some single steroids and their glucuronidated compounds, were significantly lower in the exposed workers than controls, but higher in smokers than the non-smokers + ex-smokers. Two-way analysis of variance showed a negative association between serum PCBs and both total glucuronidated 17-KS and total and glucuronidated pregnanes, and a positive association between cigarette smoking and both total and glucuronidated 17-KS. PCBs seem to act as endocrine disruptors by reducing the urinary excretion of corticosteroid hormones, particularly of the glucuronidated fraction. Cigarette smoking could boost these effects of PCBs in smokers.
\end{abstract}

Keywords: polychlorobiphenyls; corticosteroid hormones; tobacco smoke; glucuronidation; endocrine disruptor

\section{Introduction}

Polychlorobiphenyls (PCBs) are chlorinated compounds consisting of 209 possible congeners, depending on the position and number of chlorine atoms on the biphenyl. Owing to their unique properties of heat resistance and chemical stability, PCBs were ubiquitously used in many industrial and commercial applications as insulating fluids in electric transformers and other devices, and as additives in pesticides, flame-retardants, plastic materials and paints. Later, they were discovered to pose a public health hazard due to their toxic effects in humans and in animals, depending on the PCB congener, as well as their biomagnification in the food chain and persistence in the environment. For this reason, their production and use has been banned or severely restricted in most countries 
since the 1970s. Nowadays, therefore, occupational exposure to PCBs is probably limited to certain situations, such as PCBs waste disposal plants, or maintenance of contaminating appliances still in use, or else laboratories devoted to analytical determinations of the presence of these congeners [1-4]. Moreover, even though PCBs have been banned, non-occupational exposure to PCBs may still occur in the general population due to their widespread distribution in the environment and food chain.

Individual PCBs can act on different receptors including aryl hydrocarbon receptor (AhR), whose activation is one of the key events linked to carcinogenesis mediated by dioxin-like PCBs, as well as the nuclear human steroid and xenobiotic receptors (SXR), and possibly has an endocrine disruption action on the reproductive system. Previous studies have shown that PCBs can variably influence the levels of corticosteroid hormones. In fact, in some studies on experimental animals administered mixtures of PCBs by different routes, an increase in serum levels of corticosterone was reported, in others a reduction in the serum levels of corticosterone, dehydroepiandrosterone (DHEA) and DHEA sulfate (DHEA-S), while in yet other studies no effect was observed [4]. In humans, too, environmental exposure to PCBs has revealed different effects, ranging from a positive association with serum DHEA-S in children, to a reduction of the urinary metabolites of 17-ketosteroids (KS) and 17-hydroxycorticosteroids (17-OHCS), of sulfonated compounds for 17-OHCS and of the sulfonation percentage of 17-KS and 17-OHCS [5-7].

However, many other factors, either endogenous or related to lifestyle, can affect the metabolism of the corticosteroid hormones [8,9]. Among these factors, cigarette smoking has been shown to increase the plasma levels of cortisol and adrenal androgens such as androstenedione and DHEA-S, mediated by the pituitary hormones [10].

From the reports in literature, therefore, it appears that studies of the relation between the urinary excretion of corticosteroid hormones and exposure to PCBs or cigarette smoking, respectively, have elicited contrasting findings. For this reason, the aim of the present study was to investigate the influence of PCBs and a smoking habit on the urinary excretion of the metabolites of the corticosteroid hormones in subjects with occupational exposure to low concentrations of these toxicants.

\section{Materials and Methods}

\subsection{Study Subjects}

All 26 male workers still in active service at a steel factory in Taranto (Italy), who worked as electrical maintenance staff and in the past had carried out maintenance of transformers in which a mixture of PCBs (brand name "Apirolio", whose precise composition in terms of PCB congeners is not known) was used as dielectric oil, were enrolled in October to November 2009 as subjects with occupational exposure to PCBs (Exposed workers). They had carried out transformers maintenance activities during the period from 1980 to 2005. They were compared with 30 male workers at a different company in Bari, about $90 \mathrm{~km}$ from Taranto, that produces metal section bars, with no current or previous occupational exposure to PCBs (Controls), enrolled in the study for the same period.

All participants were administered, by trained medical staff in the infirmaries of the two factories, a questionnaire probing lifestyle, smoking habit, alcohol consumption, diet, hobbies that might expose to PCBs, and medical history to exclude endocrine dysfunction. All workers had been informed of the study aims and gave prior written informed consent to take part. The study was conducted in accordance with the Declaration of Helsinki.

\subsection{Blood and Urine Sampling}

On the day after filling out the questionnaire, between 7:00 a.m. and 10:00 a.m., a blood sample was taken from all the fasting exposed workers and controls, using Vacutainer SST II Advance test tubes. After blood centrifugation, the serum was distributed in two test tubes, to determine PCBs, and total cholesterol and triglycerides used to adjust the PCBs concentration levels, respectively. Immediately after blood sampling, all subjects supplied a urine sample in sterile containers for urinary 
steroids determinations. The sera used to determine PCBs and the urine samples were immediately frozen, preserved at $-20^{\circ} \mathrm{C}$ and sent to the toxicology lab; the analyses were made within 10 days of sample collection. The sera for determining total cholesterol and triglycerides were analyzed immediately after collection.

\subsection{Analytical Methods}

PCBs and lipids: The serum of all the subjects studied was examined for 33 PCB congeners $(28,31,52,74,77,81,99,101,105,114,118,123,126,128,138,146,153,156,157,167,169,170,172$, 177, 180, 183, 187, 189, 194, $196+203,201,206$, and 209) [4]. The analytical determination of PCBs was performed at the Laboratory of Occupational Hygiene and Toxicology of the Department of Experimental and Applied Medicine of the University of Brescia (Italy) using a Hewlett-Packard $6890 \mathrm{~N}$ gas-chromatograph coupled with an MSD HP 5973. For the analytical procedure, the modified version of the PCB measurement method described by Turci et al. [11] was adopted. Assessment of the intra-day precision of the method, based on five pooled human sera, evinced a coefficient of variation ranging from $3.0 \%$ to $6.6 \%$ [12]. PCB congener 30 was used for internal standard. The limit of quantification (LOQ) for each congener was equal to $0.1 \mathrm{ng} / \mathrm{mL}$. The serum PCBs, measured as $\mathrm{ng} / \mathrm{mL}$, were adjusted for the total serum lipids (TSL) and expressed as $\mathrm{ng} / \mathrm{g}$ lipids, applying the formula: TSL $(\mathrm{g} / \mathrm{L})=2.27 \times$ total cholesterol + triglycerides +0.623 [13]. The total PCBs in the exposed workers and controls were obtained by summing the results exceeding the LOQ for each congener. The TEQ content has not been calculated because the majority of dioxin-like congeners were below the LOQ and the only dioxin-like congener present in concentrations exceeding $5 \%$ of the total was the congener 118 [14].

Total cholesterol and triglycerides were determined at the Occupational Medicine Section "E. C. Vigliani" of the Interdisciplinary Department of Medicine of Bari University, Italy, using the Clinical Chemistry System ILab 300 Plus at the Instrumentation Laboratory.

Urinary steroids: Urinary steroids were measured by gas chromatography at the Laboratory of Occupational Hygiene and Toxicology of the Department of Experimental and Applied Medicine of the University of Brescia, Italy [15]. The identification of GC peaks was performed using mixtures of standard hormones obtained from Sigma Chemical Co., St Louis, MO, USA. Total urinary steroids and sulfonated portion were determined by comparing hormone concentrations obtained using enzymes capable of hydrolyzing both the glucuronidated and sulfonated compounds (S.H.P.-helix pomatia juice from the Industrie Biologique Francaise, Gennevilliers, France) with those obtained from bovine liver $\beta$-glucuronidase (Sigma Chemical Co., St Louis, MO, USA), an enzyme that has only a glucuronidasic activity [16]. The following urinary metabolites of steroids were determined: 17-OHCS: 11-deoxycortisol (THS), 11-dehydroxicortisone (THE), 11-hydroxycortisol (THF), $\alpha$-11-hydroxycortisol ( $\alpha$-THF), 11-dehydrocorticosterone (THA), corticosterone (THB), $\alpha$-corticosterone $(\alpha-\mathrm{THB})$, cortolone, $\beta$-cortolone, cortol, $\beta$-cortol; 17-KS: androsterone, DHEA, etiocholanolone, 11-hydroxyandrosterone, 11-ketoandrosterone, 11-ketoetiocholanolone; Pregnanes: pregnanediol, $\alpha$-pregnanediol, d5-pregnanediol, $\alpha$-d5-pregnanediol, pregnanetriol, d5-pregnanetriol, and pregnanetriolone.

The limit of detection of 17-OHCS was $0.02 \mathrm{mg} / \mathrm{L}$, and of $17-\mathrm{KS}$ and Pregnanes $0.01 \mathrm{mg} / \mathrm{L}$; coefficients of variations were $12.6 \%$ for $17-\mathrm{OHCS}, 8.3 \%$ for $17-\mathrm{KS}$ and $5.1 \%$ for Pregnanes. The urinary concentrations are reported as total steroids, sulfonated and glucuronidated steroids. All the urine samples showed urinary creatinine values ranging between $0.3 \mathrm{~g} / \mathrm{L}$ and $3.0 \mathrm{~g} / \mathrm{L}$, within the WHO recommended limits for biological samples to be judged acceptable [17].

\subsection{Statistical Analyses}

Statistical analyses were made using the SPSS program (version 14.0, Chicago, IL, USA). A normal distribution of all variables was verified using the Kolmogorov-Smirnov test. Not normally distributed variables were analyzed by parametric tests after logarithmic transformation or non-parametric 
Mann-Whitney test. Analyses of correlations were made with the Spearman test. The level of significance was set at $p<0.05$.

\section{Results}

There were no differences in general characteristics, lifestyle and consumption of foods with a particularly high content of PCBs between the exposed subjects and controls (Table 1).

Table 2 shows the serum concentrations of total PCBs, of congeners 118, 138, 153, 170, 180 and 187, that were present in concentrations exceeding $5 \%$ of the total, of the sum of these six congeners, as well as the percentage of each congener and of the sum of the six congeners with respect to the total PCBs, in the two groups. The concentration of total PCBs was obtained in exposed subjects versus controls, being the sum of 25 versus 11 of the 33 detectable congeners. The concentrations of total PCBs and of the various congeners and the sums of the six congeners were significantly higher in exposed subjects than controls, while the percentage sum was significantly higher in the controls. Similar findings were obtained when the results were expressed as units of nanograms per milliliter (data not shown); therefore, in the subsequent analyses only serum PCBs expressed as units of nanograms per gram of lipids was considered.

Table 1. General characteristics of the subjects exposed to PCBs and the controls.

\begin{tabular}{|c|c|c|c|c|c|c|c|c|}
\hline \multirow[t]{2}{*}{ Variable } & \multicolumn{4}{|c|}{ Exposed Subjects } & \multicolumn{4}{|c|}{ Controls } \\
\hline & $\mathrm{N}(\%)$ & Mean \pm SD & Median & Range & N (\%) & Mean \pm SD & Median & Range \\
\hline Age (years) & 26 & $37.0 \pm 6.9$ & 34.0 & $28.0-55.0$ & 30 & $38.0 \pm 8.6$ & 36.0 & $24.0-55.0$ \\
\hline Body Mass Index $\left(\mathrm{kg} / \mathrm{m}^{2}\right)$ & 26 & $26.3 \pm 3.3$ & 25.8 & $20.9-36.9$ & 30 & $25.7 \pm 3.4$ & 26.4 & $16.2-32.9$ \\
\hline Occupational exposure to PCBs (years) & 26 & $10.0 \pm 6.5$ & 8.0 & $2.0-25.0$ & - & & & \\
\hline \multicolumn{9}{|l|}{ Smoking habit } \\
\hline Smoker & $6(23.1)$ & & & & $14(46.6)$ & & & \\
\hline Non-smoker & $9(34.6)$ & & & & $8(26.7)$ & & & \\
\hline Ex-smoker (>1 year ago) & $11(42.3)$ & & & & $8(26.7)$ & & & \\
\hline \multicolumn{9}{|l|}{ Smokers (cigarettes/day) } \\
\hline $1-10$ & $5(19.2)$ & & & & $5(16.7)$ & & & \\
\hline $11-20$ & $1(3.9)$ & & & & $7(23.3)$ & & & \\
\hline$>20$ & - & & & & $2(6.6)$ & & & \\
\hline \multicolumn{9}{|l|}{ Alcohol consumption } \\
\hline Teetotal & $5(19.2)$ & & & & $1(3.3)$ & & & \\
\hline$<12$ g/day & $14(53.9)$ & & & & $17(56.7)$ & & & \\
\hline$\geqslant 12 \mathrm{~g} /$ day & $7(26.9)$ & & & & $12(40.0)$ & & & \\
\hline
\end{tabular}

Table 2. Serum concentrations of total PCBs, single congeners and the two sums of congeners expressed as units of nanograms per gram of lipids and as percentage of the total PCB, in exposed subjects and controls.

\begin{tabular}{|c|c|c|c|c|c|c|c|c|c|}
\hline \multirow{2}{*}{ PCBs } & & \multicolumn{4}{|c|}{ Exposed Subjects $(\mathrm{N}=26)$} & \multicolumn{4}{|c|}{ Controls $(\mathrm{N}=30)$} \\
\hline & & Mean \pm SD & Median & Range & $\mathrm{N}_{<\mathrm{LOQ}}{ }^{\mathrm{a}}$ & Mean \pm SD & Median & Range & $\mathrm{N}_{<\mathrm{LOQ}}{ }^{\mathrm{a}}$ \\
\hline Total & ng/g lipids ** & $1623.4 \pm 384.6$ & 259.0 & $79.3-19006.0$ & - & $166.3 \pm 114.9$ & 126.8 & $19.3-528.8$ & - \\
\hline Congener 118 & $\underset{\%^{*}}{n g}$ & $\begin{array}{c}51.5 \pm 109.5 \\
3.6 \pm 3.7\end{array}$ & $\begin{array}{c}15.5 \\
3.6\end{array}$ & $\begin{array}{l}6.3-564.1 \\
0.0-16.7\end{array}$ & 8 & $\begin{array}{c}- \\
1.5 \pm 2.9\end{array}$ & $\begin{array}{l}8.5 \\
0.0\end{array}$ & $\begin{array}{c}5.0-36.5 \\
0.0-8.3\end{array}$ & 23 \\
\hline Congener 138 & ng/g $\underset{\%}{\text { lipids }}$ & $\begin{array}{c}319.6 \pm 733.6 \\
23.2 \pm 3.8\end{array}$ & $\begin{array}{l}66.1 \\
23.8\end{array}$ & $\begin{array}{c}18.4-3666.4 \\
16.3-29.4\end{array}$ & - & $\begin{array}{c}39.5 \pm 23.2 \\
24.2 \pm 9.4\end{array}$ & $\begin{array}{l}33.6 \\
23.6\end{array}$ & $\begin{array}{c}8.6-109.4 \\
0.0-50.1\end{array}$ & 2 \\
\hline Congener 153 & ng/g lipids ${ }_{\%}^{* *}$ & $\begin{array}{c}381.0 \pm 837.8 \\
31.4 \pm 7.9\end{array}$ & $\begin{array}{l}78.4 \\
29.8\end{array}$ & $\begin{array}{c}36.7-4136.5 \\
21.3-50.1\end{array}$ & - & $\begin{array}{l}59.6 \pm 33.2 \\
40.7 \pm 13.7\end{array}$ & $\begin{array}{l}51.9 \\
36.4\end{array}$ & $\begin{array}{c}17.1-164.1 \\
30.0-100\end{array}$ & - \\
\hline Congener 170 & $\underset{\%}{\mathrm{ng} / \mathrm{g} \text { lipids * }}$ & $\begin{aligned} 121.9 & \pm 287.9 \\
5.6 & \pm 3.7\end{aligned}$ & $\begin{array}{l}22.7 \\
7.2\end{array}$ & $\begin{array}{c}7.0-1410.2 \\
0.0-9.5\end{array}$ & 7 & $\begin{array}{c}- \\
3.5 \pm 4.2\end{array}$ & $\begin{array}{l}9.7 \\
0.0\end{array}$ & $\begin{array}{l}5.8-36.5 \\
0.0-11.1\end{array}$ & 17 \\
\hline Congener 180 & $\underset{\%}{n g / g}$ lipids* & $\begin{array}{c}329.3 \pm 765.7 \\
23.4 \pm 5.1\end{array}$ & $\begin{array}{l}58.6 \\
22.3\end{array}$ & $\begin{array}{c}19.8-3744.8 \\
14.7-33.3\end{array}$ & - & $\begin{array}{l}42.9 \pm 23.4 \\
25.9 \pm 12.7\end{array}$ & $\begin{array}{l}38.0 \\
27.3\end{array}$ & $\begin{array}{l}8.2-109.4 \\
0.0-66.7\end{array}$ & 3 \\
\hline Congener 187 & ng/g lipids* & $\begin{array}{c}105.9 \pm 254.4 \\
5.1 \pm 3.4\end{array}$ & $\begin{array}{c}18.9 \\
6.1\end{array}$ & $\begin{array}{c}7.2-1269.2 \\
0.0-9.5\end{array}$ & 7 & $\begin{array}{c}- \\
3.3 \pm 4.6\end{array}$ & $\begin{array}{l}9.4 \\
0.0\end{array}$ & $\begin{array}{l}5.0-36.5 \\
0.0-13.0\end{array}$ & 19 \\
\hline $\begin{array}{l}\text { Sum of the } 6 \\
\text { congeners }\end{array}$ & ng/g lipids ${ }_{\%}^{* * *}$ & $\begin{array}{c}1309.3 \pm 2984.4 \\
92.1 \pm 9.4\end{array}$ & $\begin{array}{c}255.2 \\
96.6\end{array}$ & $\begin{array}{c}109.1-14,791.1 \\
76.4-100\end{array}$ & - & $\begin{array}{c}- \\
99.0 \pm 2.5\end{array}$ & $\begin{array}{c}150.9 \\
100\end{array}$ & $\begin{array}{c}67.7-492.3 \\
90.1-100\end{array}$ & - \\
\hline
\end{tabular}

${ }^{a}$ In the $\%$ calculations determinations lower than the LOQ were inserted in the analysis with value $0 ;{ }^{*} p<0.01$; $* * p \leqslant 0.001$. 
A significant positive correlation between the total PCBs and age was shown, both when analyzing the exposed workers $(r=0.46, p<0.05)$ and controls $(r=0.75, p<0.001)$ separately and together $(r=0.46$, $p<0.001)$. In addition, in the exposed workers total PCBs were positively correlated with the duration of exposure $(r=0.39, p<0.05)$.

In the exposed workers the urinary concentrations of total 17-KS (Table 3) and total pregnanes (Table 4) were significantly lower than in the controls, whereas the total 17-OHCS were not different between the two groups (data not shown), and so 17-OHCS were not considered in the subsequent statistical analyses. The same table also shows the different urinary 17-KS and pregnanes determined, as well as the concentrations of the glucuronidated and sulfonated fractions of both total 17-KS and pregnanes and the single steroids of the two families. All single 17-KS showed significantly lower urinary concentrations in the exposed workers than in the controls. The urinary glucuronide fraction of both total 17-KS and each different 17-KS was significantly lower in the exposed workers than the controls, whereas the sulfonate fraction showed this trend only for 11-ketoandrosterone. Of the different pregnanes, pregnanediol, $\alpha$-d5-pregnanediol, d5-pregnanediol and pregnanetriol showed significantly lower urinary concentrations in the exposed workers than the controls. The glucuronidated fraction was significantly lower in the exposed workers than the controls, not only for the total pregnanes but also for each pregnane analyzed singly and for pregnanetriolone, while the sulfonated fraction of both total pregnanes and each one analyzed singly did not show any difference between the two groups.

A correlation with the total PCBs was verified for the steroids listed in Tables 3 and 4 which were significantly lower in the exposed workers than in the controls, analyzed both separately and as a single group. A significant negative correlation only in the exposed workers and controls analyzed together was found for total 17-KS $(r=-0.28, p<0.05)$, androsterone $(r=-0.37, p<0.01)$ and the glucuronide fraction of androsterone $(r=-0.30, p<0.05)$, whereas no correlation was found when the two groups were analyzed separately.

There was no significant correlation in the exposed workers between the duration of exposure to PCBs and the different urinary 17-KS and pregnanes determined (data not shown).

Table 3. Urinary concentrations of total $17-\mathrm{KS}$ and the different steroids and their respective glucuronidated and sulfonated compounds in the exposed subjects and controls.

\begin{tabular}{|c|c|c|c|c|c|c|}
\hline 17-KS (mg/L) & \multicolumn{3}{|c|}{ Exposed Subjects $(\mathbf{N}=26)$} & \multicolumn{3}{|c|}{ Controls $(\mathrm{N}=30)$} \\
\hline Total ${ }^{\mathrm{c}}$ & $6.14 \pm 3.44$ & 5.78 & $1.58-14.68$ & $9.86 \pm 4.28$ & 8.92 & $1.38-21.85$ \\
\hline Sulfonates & $2.24 \pm 1.29$ & 2.06 & $0.15-4.83$ & $3.01 \pm 2.08$ & 2.42 & $0.18-9.21$ \\
\hline Androsterone ${ }^{b}$ & $2.51 \pm 1.31$ & 2.54 & $0.66-5.67$ & $3.66 \pm 1.84$ & 3.27 & $0.74-10.30$ \\
\hline Etiocholanolone $^{\mathrm{c}}$ & $1.62 \pm 1.02$ & 1.47 & $0.41-4.40$ & $2.74 \pm 1.26$ & 2.49 & $0.24-6.67$ \\
\hline Glucuronide $^{\mathrm{c}}$ & $1.33 \pm 1.07$ & 1.00 & $0.13-4.40$ & $2.34 \pm 1.17$ & 2.10 & $0.05-5.81$ \\
\hline Sulfonate & $0.29 \pm 0.22$ & 0.27 & $0-0.78$ & $0.40 \pm 0.47$ & 0.24 & $0-2.04$ \\
\hline DHEA $^{b}$ & $0.17 \pm 0.36$ & 0.03 & $0.01-1.63$ & $0.26 \pm 0.39$ & 0.09 & $0.01-1.74$ \\
\hline Glucuronide $^{\mathrm{b}}$ & $0.05 \pm 0.09$ & 0.02 & $<0.01-0.38$ & $0.09 \pm 0.09$ & 0.06 & $0.01-0.43$ \\
\hline 11-ketoandrosterone ${ }^{c}$ & $0.06 \pm 0.05$ & 0.04 & $0.01-0.25$ & $0.13 \pm 0.15$ & 0.09 & $0.01-0.76$ \\
\hline Glucuronide $^{\mathrm{a}}$ & $0.04 \pm 0.04$ & 0.02 & $<0.01-0.19$ & $0.07 \pm 0.07$ & 0.04 & $0.01-0.36$ \\
\hline Sulfonate ${ }^{a}$ & $0.02 \pm 0.02$ & 0.01 & 0-0.07 & $0.06 \pm 0.14$ & 0.03 & $0-0.75$ \\
\hline 11-ketoetiocholanolone ${ }^{c}$ & $0.37 \pm 0.32$ & 0.26 & $0.05-1.42$ & $0.82 \pm 0.50$ & 0.80 & $0.10-2.73$ \\
\hline Glucuronide $^{\mathrm{c}}$ & $0.27 \pm 0.31$ & 0.17 & $0.01-1.40$ & $0.66 \pm 0.50$ & 0.51 & $0.02-2.73$ \\
\hline Sulfonate & $0.09 \pm 0.12$ & 0.05 & $0-0.44$ & $0.17 \pm 0.25$ & 0.10 & $0-1.13$ \\
\hline
\end{tabular}


Table 4. Urinary concentrations of the total pregnanes and different steroids and their respective glucuronidated and sulfonated compounds in the exposed subjects and controls.

\begin{tabular}{|c|c|c|c|c|c|c|}
\hline \multirow{2}{*}{ PREGNANES (mg/L) } & \multicolumn{3}{|c|}{ Exposed Subjects $(\mathrm{N}=26)$} & \multicolumn{3}{|c|}{ Controls $(\mathrm{N}=30)$} \\
\hline & Mean \pm SD & Median & Range & Mean \pm SD & Median & Range \\
\hline Total $^{\mathrm{b}}$ & $1.66 \pm 1.26$ & 1.37 & $0.37-5.64$ & $2.58 \pm 1.14$ & 2.43 & $0.22-4.75$ \\
\hline Glucuronides $^{\text {a }}$ & $1.09 \pm 1.14$ & 0.68 & $0.09-5.26$ & $1.87 \pm 1.06$ & 1.68 & $0.09-4.35$ \\
\hline Sulfonates & $0.57 \pm 0.31$ & 0.48 & $0.15-1.24$ & $0.72 \pm 0.47$ & 0.61 & $0.02-2.19$ \\
\hline Pregnandiol ${ }^{\mathrm{b}}$ & $0.25 \pm 0.27$ & 0.20 & $0.05-1.34$ & $0.37 \pm 0.22$ & 0.32 & $0.04-0.88$ \\
\hline Glucuronide b & $0.20 \pm 0.26$ & 0.12 & $0.02-1.28$ & $0.31 \pm 0.21$ & 0.23 & $0.02-0.78$ \\
\hline Sulfonate & $0.05 \pm 0.04$ & 0.04 & $0-0.16$ & $0.06 \pm 0.05$ & 0.05 & $0-0.20$ \\
\hline$\alpha$-pregnanediol & $0.08 \pm 0.06$ & 0.06 & $0.01-0.21$ & $0.15 \pm 0.17$ & 0.10 & $0.01-0.61$ \\
\hline Glucuronide & $0.06 \pm 0.05$ & 0.04 & $0.01-0.20$ & $0.10 \pm 0.07$ & 0.08 & $0.01-0.26$ \\
\hline Sulfonate & $0.02 \pm 0.02$ & 0.01 & $0-0.06$ & $0.05 \pm 0.11$ & 0.01 & $0-0.39$ \\
\hline$\alpha$-d5-pregnanediol ${ }^{\mathrm{c}}$ & $0.27 \pm 0.32$ & 0.16 & $0.01-1.56$ & $0.56 \pm 0.40$ & 0.50 & $0.04-1.90$ \\
\hline Glucuronate $^{c}$ & $0.22 \pm 0.28$ & 0.09 & $0.01-1.29$ & $0.46 \pm 0.37$ & 0.43 & $0.02-1.90$ \\
\hline Sulfonate & $0.05 \pm 0.06$ & 0.04 & $0-0.27$ & $0.10 \pm 0.16$ & 0.05 & $0-0.77$ \\
\hline d5-pregnanediol a & $0.10 \pm 0.08$ & 0.08 & $0.01-0.29$ & $0.15 \pm 0.08$ & 0.14 & $0.01-0.33$ \\
\hline Glucuronide ${ }^{\mathrm{b}}$ & $0.04 \pm 0.04$ & 0.03 & $<0.01-0.15$ & $0.08 \pm 0.05$ & 0.08 & $0.01-0.19$ \\
\hline Sulfonate & $0.06 \pm 0.05$ & 0.03 & $0-0.15$ & $0.07 \pm 0.05$ & 0.06 & $0-0.22$ \\
\hline Pregnanetriol $^{\mathrm{b}}$ & $0.69 \pm 0.46$ & 0.54 & $0.18-1.88$ & $1.10 \pm 0.48$ & 1.08 & $0.09-2.26$ \\
\hline Glucuronide $^{c}$ & $0.40 \pm 0.44$ & 0.23 & $0.02-1.88$ & $0.74 \pm 0.49$ & 0.65 & $0.01-1.98$ \\
\hline Sulfonate & $0.29 \pm 0.19$ & 0.25 & $0-0.77$ & $0.35 \pm 0.27$ & 0.29 & $0-1.02$ \\
\hline d5-pregnanetriol & $0.10 \pm 0.17$ & 0.05 & $0.01-0.80$ & $0.10 \pm 0.08$ & 0.07 & $0.01-0.29$ \\
\hline Glucuronide & $0.10 \pm 0.17$ & 0.05 & $0.01-0.80$ & $0.10 \pm 0.08$ & 0.07 & $0.01-0.29$ \\
\hline Sulfonate & - & - & - & - & - & $0-0.03$ \\
\hline Pregnanetriolone & $0.19 \pm 0.16$ & 0.17 & $0.01-0.55$ & $0.22 \pm 0.15$ & 0.19 & $0.02-0.72$ \\
\hline Glucuronide ${ }^{a}$ & $0.08 \pm 0.10$ & 0.04 & $0.01-0.45$ & $0.12 \pm 0.13$ & 0.09 & $0.01-0.72$ \\
\hline Sulfonate & $0.11 \pm 0.11$ & 0.10 & $0-0.38$ & $0.10 \pm 0.07$ & 0.10 & $0-0.22$ \\
\hline
\end{tabular}

To assess the effect of a smoking habit on the excretion of urinary steroids, the exposed workers and controls were subdivided into two groups, smokers versus non-smokers + ex-smokers. Compared with non-smokers + ex-smokers, smokers had significantly higher urinary concentrations of total 17-KS, androsterone, etiocholanolone, 11-ketoandrosterone, 11-ketoetiocholanolone and their glucuronidated compounds, whereas for DHEA and 11-hydroxyandrosterone only the glucuronidated fraction was significantly higher (Supplementary Table S1). Smokers also showed significantly higher levels of total pregnanes, pregnanediol, $\alpha$-d5-pregnanediol and d5-pregnanediol and their glucuronidated fractions (Supplementary Table S2). Instead, no difference was found between smokers and non-smokers + ex-smokers for the urinary excretion of total 17-OHCS and the metabolites analyzed singly.

A correlation among 17-KS, pregnanes, those glucuronidated fractions found to be significantly higher in the smokers and total PCBs was also analyzed in the smokers and non-smokers + ex-smokers. In the smoker group (Table 5), no correlation was found between total PCBs and the different urinary steroids. By contrast, in the non-smokers + ex-smokers there was a significant negative correlation between total PCBs and total 17-KS, androsterone, etiocholanolone, total pregnanes, d5-pregnanediol and their glucuronidated fractions, except for etiocholanolone. In view of the opposite action exerted by total PCBs and cigarette smoking on the urinary excretion of 17-KS, pregnanes and their glucuronidated fractions, two-way analysis of variance was applied to assess the role of these two risk factors. Only those urinary steroids and their glucuronidated fractions found to be significantly reduced in the exposed workers compared to the controls were analyzed (Tables 3 and 4), and those significantly increased in the smokers compared to the non-smokers + ex-smokers. A significant association resulted for the 17-KS between exposure both to PCBs and to cigarette smoking and glucuronidated 17-KS, etiocholanolone and etiocholanolone glucuronide, and between cigarette smoking and total 17-KS, androsterone, 11-ketoetiocholanolone and 11-ketoetiocholanolone glucuronide (Table 6). For the pregnanes, a significant association was found between exposure to PCBs and total pregnanes, pregnanediol and the glucuronidated compound and $\mathrm{d} 5$-pregnanediol, and between cigarette smoking and $\alpha$-d5-pregnanediol, d5-pregnanediol glucuronide, pregnanetriol and pregnanetriol glucuronide (Table 7). 
Table 5. Significant correlations among urinary 17-KS, pregnanes and their respective glucuronidated compounds and total PCBs in smokers and non-smokers + ex-smokers, analyzed separately.

\begin{tabular}{ccc}
\hline Hormones & \multicolumn{2}{c}{ Total PCBs } \\
\cline { 2 - 3 } & Smokers $\mathbf{N}=\mathbf{2 0})$ & Non-Smokers + Ex-Smokers (N = 36) \\
\hline Total 17-KS & 0.01 & $-0.45^{\mathrm{b}}$ \\
Glucuronides & 0.06 & $-0.40^{\mathrm{a}}$ \\
Androsterone & -0.15 & $-0.52^{\mathrm{c}}$ \\
Glucuronide & -0.07 & $-0.40^{\mathrm{a}}$ \\
Etiocholanolone & -0.05 & $-0.36^{\mathrm{a}}$ \\
DHEA & 0.11 & $-0.44^{\mathrm{b}}$ \\
Glucuronide & 0.07 & $-0.34^{\mathrm{a}}$ \\
\hline Total Pregnanes & 0.12 & $-0.38^{\mathrm{a}}$ \\
Glucuronides & 0.14 & $-0.33^{\mathrm{a}}$ \\
d5-pregnanediol & 0.23 & $-0.35^{\mathrm{a}}$ \\
Glucuronide & 0.13 & $-0.35^{\mathrm{a}}$ \\
\hline
\end{tabular}

17-ketosteroids; DHEA: dehydroepiandrosterone; ${ }^{\mathrm{a}} p<0.05{ }^{\mathrm{b}} p \leqslant 0.01{ }^{\mathrm{c}} p=0.001$. 
Table 6. Influence of exposure to PCBs and cigarette smoking on total urinary 17-KS and 17-KS glucuronides assessed by two-way analysis of variance.

\begin{tabular}{|c|c|c|c|c|c|c|c|c|c|c|}
\hline \multirow{2}{*}{ Hormones } & \multirow{2}{*}{$\begin{array}{l}\text { Exposure to } \\
\text { PCBs }\end{array}$} & \multicolumn{4}{|c|}{ Smokers } & \multicolumn{4}{|c|}{ Non-Smokers + Ex-Smokers } & \multirow[b]{2}{*}{ ANOVA } \\
\hline & & $\mathbf{N}$ & Mean \pm SD & Median & Range & $\mathbf{N}$ & Mean \pm SD & Median & Range & \\
\hline Total 17-KS & $\begin{array}{l}\text { Yes } \\
\text { No }\end{array}$ & $\begin{array}{c}6 \\
14\end{array}$ & $\begin{array}{c}7.97 \pm 5.74 \\
10.59 \pm 3.69\end{array}$ & $\begin{array}{l}6.52 \\
9.63\end{array}$ & $\begin{array}{l}2.15-14.68 \\
4.80-17.49\end{array}$ & $\begin{array}{l}20 \\
16\end{array}$ & $\begin{array}{l}5.59 \pm 2.35 \\
9.23 \pm 4.75\end{array}$ & $\begin{array}{l}5.78 \\
8.50\end{array}$ & $\begin{array}{l}1.58-10.58 \\
1.38-21.85\end{array}$ & $\begin{array}{l}\text { Model: } p \leqslant 0.01 \\
\text { Smoke: } p \leqslant 0.01 \\
\text { PCB: NS }\end{array}$ \\
\hline Glucuronides & $\begin{array}{l}\text { Yes } \\
\text { No }\end{array}$ & $\begin{array}{c}6 \\
14\end{array}$ & $\begin{array}{l}5.71 \pm 5.06 \\
7.98 \pm 3.50\end{array}$ & $\begin{array}{l}3.47 \\
6.93\end{array}$ & $\begin{array}{l}1.24-13.81 \\
3.43-15.48\end{array}$ & $\begin{array}{l}20 \\
16\end{array}$ & $\begin{array}{l}3.35 \pm 2.48 \\
5.86 \pm 3.92\end{array}$ & $\begin{array}{l}2.97 \\
4.67\end{array}$ & $\begin{array}{c}0.25-9.17 \\
0.16-17.68\end{array}$ & $\begin{array}{l}\text { Model: } p \leqslant 0.01 \\
\text { Smoke: } p<0.05 \\
\text { PCB: } p<0.05\end{array}$ \\
\hline Androsterone & $\begin{array}{l}\text { Yes } \\
\text { No }\end{array}$ & $\begin{array}{c}6 \\
14\end{array}$ & $\begin{array}{l}2.88 \pm 2.12 \\
3.99 \pm 1.41\end{array}$ & $\begin{array}{l}2.55 \\
3.61\end{array}$ & $\begin{array}{l}0.66-5.67 \\
1.90-6.09\end{array}$ & $\begin{array}{l}20 \\
16\end{array}$ & $\begin{array}{l}2.40 \pm 1.01 \\
3.37 \pm 2.15\end{array}$ & $\begin{array}{l}2.54 \\
2.82\end{array}$ & $\begin{array}{c}0.88-4.71 \\
0.74-10.30\end{array}$ & $\begin{array}{l}\text { Model: } p<0.05 \\
\text { Smoke: } p<0.05 \\
\text { PCB: NS }\end{array}$ \\
\hline Etiocholanolone & $\begin{array}{l}\text { Yes } \\
\text { No }\end{array}$ & $\begin{array}{c}6 \\
14\end{array}$ & $\begin{array}{l}2.17 \pm 1.63 \\
3.34 \pm 1.43 \\
\end{array}$ & $\begin{array}{l}1.69 \\
3.22\end{array}$ & $\begin{array}{l}0.63-4.40 \\
1.11-6.67\end{array}$ & $\begin{array}{l}20 \\
16\end{array}$ & $\begin{array}{l}1.46 \pm 0.75 \\
2.21 \pm 0.82\end{array}$ & $\begin{array}{l}1.48 \\
2.34\end{array}$ & $\begin{array}{l}0.41-3.09 \\
0.24-3.32\end{array}$ & $\begin{array}{l}\text { Model: } p<0.001 \\
\text { Smoke: } p \leqslant 0.01 \\
\text { PCB: } p \leqslant 0.01\end{array}$ \\
\hline Glucuronide & $\begin{array}{l}\text { Yes } \\
\text { No }\end{array}$ & $\begin{array}{c}6 \\
14\end{array}$ & $\begin{array}{l}2.02 \pm 1.60 \\
2.93 \pm 1.28\end{array}$ & $\begin{array}{l}1.46 \\
2.67\end{array}$ & $\begin{array}{l}0.48-4.40 \\
0.99-5.81\end{array}$ & $\begin{array}{l}20 \\
16\end{array}$ & $\begin{array}{l}1.12 \pm 0.79 \\
1.82 \pm 0.77\end{array}$ & $\begin{array}{l}0.97 \\
1.93\end{array}$ & $\begin{array}{l}0.13-3.09 \\
0.05-3.07\end{array}$ & $\begin{array}{l}\text { Model: } p<0.001 \\
\text { Smoke: } p<0.05 \\
\text { PCB: } p \leqslant 0.01\end{array}$ \\
\hline 11-hydroxyandrosterone & $\begin{array}{l}\text { Yes } \\
\text { No }\end{array}$ & $\begin{array}{c}6 \\
14\end{array}$ & $\begin{array}{l}1.84 \pm 1.23 \\
2.07 \pm 0.68\end{array}$ & $\begin{array}{l}1.82 \\
2.14\end{array}$ & $\begin{array}{l}0.40-3.74 \\
0.83-3.18\end{array}$ & $\begin{array}{l}20 \\
16\end{array}$ & $\begin{array}{l}1.32 \pm 0.56 \\
2.58 \pm 2.11\end{array}$ & $\begin{array}{l}1.42 \\
2.22\end{array}$ & $\begin{array}{l}0.17-2.57 \\
0.28-8.84\end{array}$ & $\begin{array}{l}\text { Model: } p<0.05 \\
\text { Smoke: NS } \\
\text { PCB: NS }\end{array}$ \\
\hline 11-ketoetiocholanolone & $\begin{array}{l}\text { Yes } \\
\text { No }\end{array}$ & $\begin{array}{c}6 \\
14\end{array}$ & $\begin{array}{l}0.59 \pm 0.51 \\
0.82 \pm 0.32\end{array}$ & $\begin{array}{l}0.51 \\
0.87\end{array}$ & $\begin{array}{l}0.09-1.42 \\
0.29-1.32\end{array}$ & $\begin{array}{l}20 \\
16\end{array}$ & $\begin{array}{l}0.30 \pm 0.22 \\
0.82 \pm 0.63\end{array}$ & $\begin{array}{l}0.26 \\
0.74\end{array}$ & $\begin{array}{l}0.05-0.87 \\
0.10-2.73\end{array}$ & $\begin{array}{l}\text { Model: } p \leqslant 0.01 \\
\text { Smoke: } p \leqslant 0.01 \\
\text { PCB: NS }\end{array}$ \\
\hline Glucuronide & $\begin{array}{l}\text { Yes } \\
\text { No }\end{array}$ & $\begin{array}{c}6 \\
14\end{array}$ & $\begin{array}{l}0.46 \pm 0.55 \\
0.69 \pm 0.28\end{array}$ & $\begin{array}{l}0.17 \\
0.59\end{array}$ & $\begin{array}{l}0.06-1.40 \\
0.29-1.32\end{array}$ & $\begin{array}{l}20 \\
16\end{array}$ & $\begin{array}{l}0.22 \pm 0.18 \\
0.63 \pm 0.64\end{array}$ & $\begin{array}{l}0.18 \\
0.47\end{array}$ & $\begin{array}{l}0.01-0.71 \\
0.02-2.73\end{array}$ & $\begin{array}{l}\text { Model: } p \leqslant 0.01 \\
\text { Smoke: } p<0.05 \\
\text { PCB: NS }\end{array}$ \\
\hline
\end{tabular}


Table 7. Influence of exposure to PCBs and cigarette smoke on total urinary pregnanes and glucuronides assessed by two-way analysis of variance.

\begin{tabular}{|c|c|c|c|c|c|c|c|c|c|c|}
\hline \multirow{2}{*}{ Hormones } & \multirow{2}{*}{$\begin{array}{c}\text { Exposure to } \\
\text { PCBs }\end{array}$} & \multicolumn{4}{|c|}{ Smokers } & \multicolumn{4}{|c|}{ Non-Smokers + Ex-Smokers } & \multirow[b]{2}{*}{ ANOVA } \\
\hline & & $\mathbf{N}$ & Mean \pm SD & Median & Range & $\mathbf{N}$ & Mean \pm SD & Median & Range & \\
\hline \multirow{2}{*}{ Total Pregnanes } & Yes & 6 & $2.47 \pm 2.05$ & 1.82 & $0.69-5.64$ & 20 & $1.42 \pm 0.84$ & 1.37 & $0.37-3.42$ & \multirow{2}{*}{$\begin{array}{l}\text { Model: } p \leqslant 0.01 \\
\text { Smoke: NS } \\
\text { PCB: } p<0.05\end{array}$} \\
\hline & No & 14 & $2.84 \pm 1.11$ & 2.75 & $1.29-4.75$ & 16 & $2.36 \pm 1.15$ & 2.24 & $0.22-4.66$ & \\
\hline \multirow{2}{*}{ Glucuronides } & Yes & 6 & $1.89 \pm 1.89$ & 1.06 & $0.50-5.26$ & 20 & $0.85 \pm 0.72$ & 0.67 & $0.09-2.49$ & \multirow{2}{*}{$\begin{array}{l}\text { Model: } p \leqslant 0.01 \\
\text { Smoke: NS } \\
\text { PCB: } p<0.05\end{array}$} \\
\hline & No & 14 & $2.15 \pm 1.05$ & 1.76 & $0.85-4.33$ & 16 & $1.62 \pm 1.03$ & 1.56 & $0.09-4.35$ & \\
\hline \multirow{2}{*}{ Pregnanediol } & Yes & 6 & $0.49 \pm 0.48$ & 0.32 & $0.12-1.34$ & 20 & $0.18 \pm 0.10$ & 0.20 & $0.05-0.37$ & \multirow{2}{*}{$\begin{array}{l}\text { Model: } p \leqslant 0.01 \\
\text { Smoke: NS } \\
\text { PCB: } p \leqslant 0.01\end{array}$} \\
\hline & No & 14 & $0.44 \pm 0.25$ & 0.32 & $0.188-0.88$ & 16 & $0.31 \pm 0.18$ & 0.30 & $0.04-0.70$ & \\
\hline \multirow{2}{*}{ Glucuronide } & Yes & 6 & $0.45+0.47$ & 0.28 & $0.10-1.28$ & 20 & $0.13+0.08$ & 0.12 & $0.02-0.28$ & \multirow{2}{*}{$\begin{array}{l}\text { Model: } p \leqslant 0.01 \\
\text { Smoke: NS } \\
\text { PCB: } p<0.001\end{array}$} \\
\hline & No & 14 & $0.38 \pm 0.23$ & 0.29 & $0.14-0.78$ & 16 & $0.25 \pm 0.16$ & 0.20 & $0.02-0.62$ & \\
\hline \multirow[b]{2}{*}{$\alpha$-d5-pregnanediol } & Yes & 6 & $0.41 \pm 0.24$ & 0.37 & $0.06-0.74$ & 20 & $0.23 \pm 0.34$ & 0.11 & $0.01-1.56$ & \multirow{2}{*}{$\begin{array}{l}\text { Model: } p<0.05 \\
\text { Smoke: } p<0.05 \\
\text { PCB: NS }\end{array}$} \\
\hline & No & 14 & $0.61 \pm 0.46$ & 0.51 & $0.13-1.90$ & 16 & $0.51 \pm 0.34$ & 0.46 & $0.04-1.24$ & \\
\hline \multirow[b]{2}{*}{ d5-pregnanediol } & Yes & 6 & $0.15+0.12$ & 0.13 & $0.03-0.29$ & 20 & $0.08+0.06$ & 0.07 & $0.01-0.18$ & \multirow{2}{*}{$\begin{array}{l}\text { Model: } p<0.05 \\
\text { Smoke: NS } \\
\text { PCB: } p<0.05\end{array}$} \\
\hline & No & 14 & $0.16 \pm 0.07$ & 0.15 & $0.07-0.32$ & 16 & $0.14 \pm 0.09$ & 0.14 & $0.01-0.33$ & \\
\hline \multirow[b]{2}{*}{ Glucuronide } & Yes & 6 & & 0.05 & $0.01-0.15$ & 20 & & 002 & $<001-013$ & \multirow{2}{*}{$\begin{array}{l}\text { Model: } p<0.05 \\
\text { Smoke: } p<0.05 \\
\text { PCB: NS }\end{array}$} \\
\hline & No & 14 & $0.09 \pm 0.04$ & 0.08 & $0.01-0.19$ & 16 & $0.08 \pm 0.05$ & 0.07 & $0.01-0.18$ & \\
\hline \multirow{3}{*}{ Pregnanetriol } & & & & & & & & & & \multirow{3}{*}{$\begin{array}{l}\text { Model: } p \leqslant 0.01 \\
\text { Smoke: } p<0.05 \\
\text { PCB: NS }\end{array}$} \\
\hline & Yes & 6 & $0.85 \pm 0.68$ & 0.69 & $0.18-1.88$ & 20 & $0.64 \pm 0.38$ & 0.55 & $0.20-0.52$ & \\
\hline & No & 14 & $1.21 \pm 0.50$ & 1.23 & $0.37-2.26$ & 16 & $1.00 \pm 0.46$ & 0.92 & $0.09-1.65$ & \\
\hline \multirow[b]{2}{*}{ Glucuronide } & Yes & 6 & $0.59 \pm 0.69$ & 0.27 & $0.14-1.88$ & 20 & $0.34 \pm 0.34$ & 0.23 & $0.02-1.43$ & \multirow{2}{*}{$\begin{array}{l}\text { Model: } p<0.05 \\
\text { Smoke: } p<0.05 \\
\text { PCB: NS }\end{array}$} \\
\hline & No & 14 & $0.88 \pm 0.53$ & 0.74 & $0.22-1.98$ & 16 & $0.62 \pm 0.43$ & 0.55 & $0.01-1.65$ & \\
\hline
\end{tabular}




\section{Discussion}

The study shows that exposure to low concentrations of PCBs might be associated with a reduced urinary excretion of 17-KS and pregnanes, both total and the single steroid hormones, and of their glucuronidated fractions, whereas cigarette smoking is associated with an increased urinary excretion. Neither of the risk factors affects the urinary excretion of 17-OHCS.

Exposed worker group showed concentrations of total PCBs higher than controls and a partially different profile in terms of the congeners. In fact, the percentage sum of the six congeners exceeding $5 \%$ of the total was $100 \%$ as median value in the controls and 96.6 in the exposed worker group. These results, however, suggest a different origin of exposure to PCBs for the two groups, although the differences are not so evident because of the time from the last exposure. Total PCBs are also positively correlated with age, and in the exposed workers group, with the years of exposure to PCBs, in agreement with data in the literature reporting a tendency of these compounds to accumulate in the organism with an increasing duration of exposure [4,18].

The reduced urinary excretion of total 17-KS observed in our study is in agreement with the report by Romeo et al. [6] in subjects of the general population resident in urban areas near an industrial plant producing PCBs. These subjects had a mean serum concentration of total PCBs of $61.9 \mathrm{ng} / \mathrm{mL}$ calculated on 24 congeners, higher than the $10.04 \mathrm{ng} / \mathrm{mL}$, calculated on 33 congeners, observed in our workers exposed to PCBs. These Authors also found a reduced urinary excretion of total 17-OHCS and their sulfonated compound but no difference in the urinary excretion of the total pregnanes and their sulfonated compounds. They did not study the glucuronidated compounds of any of these three classes of urinary steroids. Our study, therefore, even confirming the possible effect of PCBs as endocrine disruptors of the endogenous steroid hormone homeostasis, seems to show some differences from the findings of Romeo et al. Such differences lead us to hypothesize that PCBs target and mode of action could be, at least partially, influenced by the exposure level and/or the pattern of congeners exposure, the latter depending on occupational or environmental exposure.

Glucuronidation and sulfonation are catalyzed by the UDP-glucuronosyltransferase (UGT) enzyme family, in the presence of the cofactor uridine diphosfate-glucuronide (UDPglucuronide), and by the sulfotransferase (SULT) enzyme family in the presence of the cofactor $3^{\prime}$-phosphoadenosine- $5^{\prime}$ -phosphosulfate (PAPS). Apart from the steroid hormones, these enzymes also have various endogenous and exogenous chemical compounds as substrate, including PCBs. At the hepatic level the latter can undergo phase I and II metabolic reactions, as confirmed both in vitro and in experimental animals [19-22]. In particular, after hydroxylation by the P450 cytochromes, congeners with a lower degree of chlorination can be glucuronidated or sulfonated and so eliminated in bile and, to a lesser extent, in urine. Otherwise, they can remain in the blood, or be newly hydroxylated to quinones. By contrast, congeners with greater chlorine content are less susceptible to metabolic reactions and so persist in biological tissues.

The reduction only of the glucuronidated fraction of 17-KS and pregnanes, observed in our exposed workers, suggests that this could have been determined by the PCBs through enzymatic inhibition during the glucuronidation phase. This inhibition could depend on a reduction of UDP glucuronide, that is therefore subtracted from the glucuronidation reactions of the other compounds, as demonstrated by Chan et al. [23] in rat hepatocytes treated in vitro with a mixture of PCBs (congeners $28,77,105,126$, and 153). However, other mechanisms could underlie the inhibitory effect. Highly chlorinated PCB congeners, for instance, and especially PCB 153, are antagonists of SXR and so can inhibit the genetic induction of the phase I and II metabolism enzymes that are physiologically activated by the steroid hormones [24]. This antagonistic effect exerted by PCBs on the SXR receptor could also negatively modulate the induction of the microsomial enzymes [24,25], as described in the literature for the dioxin-like PCBs, mediated by the activation of the AhR [26-28]. Finally, a possible direct effect of PCBs on the hepatic enzymes of the phase II metabolism has been described, inhibiting both the glucuronidation and sulfonation of some steroid and xenobiotic hormones $[1,2,21,28]$. 
In our study, no significant reduction of the urinary excretion of the sulfonated steroid hormones was observed in the exposed workers. An inhibition of the SULT, and in particular of the hSULT2A1 isoform, has recently been described in some in vitro studies that suggested a possible non competitive inhibition mechanism exerted both by a mixture of $\mathrm{OH}-\mathrm{PCB}$, probably depending on the availability of the PAPS cofactor, and of PCB quinones, highly oxidating metabolites of the PCBs [29-32]. However, Van den Hurk et al. [33] demonstrated, at the level of the bowel mucosa of the catfish, that hydroxylated PCBs inhibit the UGT enzymes to a greater extent than the SULT. They pointed out that the SULT are enzymes with a high affinity and low capacity while the UGT have low affinity and high capacity. Further study seems necessary to clarify whether the effects on the sulfonation of the steroid hormones by the PCB metabolites observed in vitro are also present in man as a consequence of environmental or occupational exposure to low concentrations of PCBs, and whether these can cause an effect of endocrine disruption.

Our results demonstrated, in smokers, a higher urinary excretion of the total 17-KS and total pregnanes, their glucuronidated fractions and of some of the single steroid hormones, in agreement with previous studies [34,35]. This effect could be due either to the toxic action of nicotine or to increased stress effects on the organism induced by the smoking habit [10]. Moreover, cigarette smoke could enhance the expression of several P450 cytochromes [36]. In our study, a smoking habit seemed to have a confounding effect on the inhibition by PCBs of the urinary excretion of 17-KS and pregnanes and, in particular, of their glucuronidated fractions, as shown by the significant correlation between these steroids and the total PCBs in the non-smoker + ex-smoker group but not in the smoker group. This negative effect of PCBs and positive effect of cigarette smoking on the urinary excretion of the steroids was confirmed by two-way analysis of variance.

This study presents some limits that should be considered, including small sample size and the fact that, for company organization reasons, the urinary levels of the corticosteroid hormones were measured on urine spot samples and not on $24 \mathrm{~h}$ urine collection samples. Moreover, these determinations were not associated with evaluation of the serum levels.

\section{Conclusions}

Our results clearly indicate that PCBs could have an effect as endocrine disruptors by reducing the urinary excretion of the steroid hormones through inhibiting their glucuronidation and/or through other effects on their metabolic biotransformation pathways. This could likely result in their persistence in the organism. Cigarette smoking, in turn, by stimulating the production of these hormones, could boost their serum concentrations. Together with the reduced urinary excretion caused by the PCBs, this could foster increased biological effects in the organism. To clarify this interaction, and whether causes clinical effects in exposed workers, it could be useful to determine both the urinary and the serum concentrations of the steroids at the same time, bearing in mind that these glucuronidation mechanisms are shared by many other environmental pollutants.

Supplementary Materials: The following are available online at www.mdpi.com/1660-4601/13/4/360/s1, Table S1: Urinary concentrations of 17-KS in exposed subjects and controls subdivided by smoking habit, Table S2: Urinary concentrations of pregnanes in exposed subjects and controls subdivided by smoking habit.

Author Contributions: Maria Nicolà D'Errico and Piero Lovreglio designed the study, with the contribution of Leonardo Soleo. Maria Nicolà D'Errico and Piero Lovreglio recruited the participants and collected the data. Pietro Apostoli performed analyses of PCB. Ignazio Drago performed statistical analyses of the data with the contribution of Piero Lovreglio. Maria Nicolà D'Errico and Piero Lovreglio prepared the manuscript draft with important intellectual input from all the other authors. Leonardo Soleo and Pietro Apostoli supervised the study. All authors participated in critical revision of the manuscript and read and approved the final version

Conflicts of Interest: The authors declare no conflict of interest.

\section{References}

1. ATSDR. Toxicological Profile for Polychlorinated Biphenyls (PCBs). Available online: http://www.atsdr. cdc.gov/toxprofiles/tp17.pdf (accessed on 15 January 2016). 
2. Diamanti-Kandarakis, E.; Bourguignon, J.P.; Giudice, L.C.; Hauser, R.; Prins, G.S.; Soto, A.M.; Zoeller, R.T.; Gore, A.C. Endocrine-disrupting chemicals: An endocrine society scientific statement. Endocr. Rev. 2009, 30, 293-342. [CrossRef] [PubMed]

3. Erickson, M.D. Introduction: PCBs properties, uses, occurrence and regulatory history. In PCBs. Recent Advances in Environmental Toxicology and Health Effects; Robertson, L.W., Hansen, L.G., Eds.; The University Press of Kentucky: Lexington, KY, USA, 2001.

4. IPCS. Concise International Chemical Assessment Document 55. Polychlorinated Biphenyls: Human Health Aspects. Available online: http://www.who.int/ipcs/publications/cicad/en/cicad55.pdf (accessed on 15 January 2016).

5. Rennert, A.; Wittsiepe, J.; Kasper-Sonnenberg, M.; Binder, G.; Fürst, P.; Cramer, C.; Krämer, U.; Wilhelm, M. Prenatal and early life exposure to polychlorinated dibenzo-p-dioxins, dibenzofurans and biphenyls may influence dehydroepiandrosterone sulfate levels at prepubertal age: Results from the Duisburg birth cohort study. J. Toxicol. Environ. Health 2012, 75, 1232-1240. [CrossRef] [PubMed]

6. Romeo, L.; Catalani, S.; Pasini, F.; Bergonzi, R.; Perbellini, L.; Apostoli, P. Xenobiotic action on steroid hormone synthesis and sulfonation the example of lead and polychlorinated biphenyls. Int. Arch. Occup. Environ. Health 2009, 82, 557-564. [CrossRef] [PubMed]

7. Turyk, M.E.; Anderson, H.A.; Freels, S.; Chatterton, R., Jr.; Needham, L.L.; Patterson, D.G., Jr.; Steenport, D.N.; Knobeloch, L.; Imm, P.; Persky, V.W. Great Lakes Consortium Associations of organochlorines with endogenous hormones in male Great Lakes fish consumers and nonconsumers. Environ. Res. 2006, 102, 299-307. [CrossRef] [PubMed]

8. Sarkola, T.; Eriksson, C.J. Testosterone increases in men after a low dose of alcohol. Alcohol Clin. Exp. Res. 2003, 27, 682-685. [CrossRef] [PubMed]

9. Waring, R.H.; Ayers, S.; Gescher, A.J.; Glatt, H.R.; Meinl, W.; Jarratt, P.; Kirk, C.J.; Pettitt, T.; Rea, D.; Harris, R.M. Phytoestrogens and xenoestrogens: The contribution of diet and environment to endocrine disruption. J. Steroid. Biochem. Mol. Biol. 2008, 108, 213-220. [CrossRef] [PubMed]

10. Kapoor, D.; Jones, T.H. Smoking and hormones in health and endocrine disorders. Eur. J. Endocrinol. 2005, 152, 491-499. [CrossRef] [PubMed]

11. Turci, R.; Angeleri, F.; Minoia, C. A rapid screening method for routine congener-specific analysis of polychlorinated biphenyls in human serum by high-resolution gas chromatography with mass spectrometric detection. Rapid Commun. Mass Spectrom. 2002, 16, 1957-1964. [CrossRef] [PubMed]

12. Apostoli, P.; Magoni, M.; Bergonzi, R.; Carasi, S.; Indelicato, A.; Scarcella, C.; Donato, F. Assessment of reference values for polychlorinated biphenyl concentration in human blood. Chemosphere 2005, 61, 413-421. [CrossRef] [PubMed]

13. Phillips, D.L.; Pirkle, J.L.; Burse, V.W.; Bernert, J.T., Jr.; Henderson, L.O.; Needham, L.L. Chlorinated hydrocarbon levels in human serum: Effects of fasting and feeding. Arch. Environ. Contam. Toxicol. 1989, 18, 495-500. [CrossRef] [PubMed]

14. D’Errico, M.N.; de Tullio, G.; di Gioacchino, M.; Lovreglio, P.; Basso, A.; Drago, I.; Serra, R.; Apostoli, P.; Vacca, A.; Soleo, L. Immune effects of polychlorinated biphenyls, smoking and alcohol. Int. J. Immunopathol. Pharmacol. 2012, 25, 1041-1054. [PubMed]

15. Faccini, G.; Bertozzo, L.; Pasini, F.; Gerani, C.; Martinelli, C.; Pasqualini, E. A new simplified method for the determination of 23 urinary steroids for the assessment of gonadic-adrenal functionality. G. Ital. Chim. Clin. 1995, 20, 305-316.

16. Apostoli, P.; Romeo, L.; Peroni, E.; Ferioli, A.; Ferrari, S.; Pasini, F.; Aprili, F. Steroid hormone sulphation in lead workers. Br. J. Ind. Med. 1989, 46, 204-208. [CrossRef] [PubMed]

17. WHO. Biological Monitoring of Chemical Exposure in the Workplace; World Health Organization: Geneva, Switzerland, 1996; Volume 1.

18. Moon, H.B.; Kim, H.S.; Choi, M.; Yu, J.; Choi, H.G. Human health risk of polychlorinated biphenyls and organochlorine pesticides resulting from seafood consumption in South Korea, 2005-2007. Food Chem. Toxicol. 2009, 47, 1819-1825. [CrossRef] [PubMed]

19. Daidoji, T.; Gozu, K.; Iwano, H.; Inoue, H.; Yokota, H. UDP-glucuronosyltransferase isoforms catalyzing glucuronidation of hydroxy-polychlorinated biphenyls in rat. Drug Metab. Dispos. 2005, 33, 1466-1476. [CrossRef] [PubMed] 
20. James, M.O. Polychlorinated biphenyls: Metabolism and metabolites. In PCBs. Recent Advances in Environmental Toxicology and Health Effects; Robertson, L.W., Hansen, L.G., Eds.; The University Press of Kentucky: Lexington, KY, USA, 2001; pp. 35-46.

21. James, M.O.; Sacco, J.C.; Faux, L.R. Effects of food natural products on the biotransformation of PCBs. Environ. Toxicol. Pharmacol. 2008, 25, 211-217. [CrossRef] [PubMed]

22. Tampal, N.; Lehmler, H.J.; Espandiari, P.; Malmberg, T.; Robertson, L.W. Glucuronidation of hydroxylated polychlorinated biphenyls (PCBs). Chem. Res. Toxicol. 2002, 15, 1259-1266. [CrossRef] [PubMed]

23. Chan, T.S.; Wilson, J.X.; Selliah, S.; Bilodeau, M.; Zwingmann, C.; Poon, R.; O’Brien, P.J. Organochlorines inhibit acetaminophen glucuronidation by redirecting UDP-glucuronic acid towards the D-glucuronate pathway. Toxicol. Appl. Pharmacol. 2008, 232, 456-462. [CrossRef] [PubMed]

24. Tabb, M.M.; Kholodovych, V.; Grün, F.; Zhou, C.; Welsh, W.J.; Blumberg, B. Highly chlorinated PCBs inhibit the human xenobiotic response mediated by the steroid and xenobiotic receptor (SXR). Environ. Health Perspect. 2004, 112, 163-169. [CrossRef] [PubMed]

25. Lauby-Secretan, B.; Loomis, D.; Grosse, Y.; El Ghissassi, F.; Bouvard, V.; Benbrahim-Tallaa, L.; Guha, N.; Baan, R.; Mattock, H.; Straif, K.; et al. Carcinogenicity of polychlorinated biphenyls and polybrominated biphenyls. Lancet Oncol. 2013, 14, 287-288. [CrossRef]

26. Nimmagadda, D.; Cherala, G.; Ghatta, S. Cytosolic sulfotransferases. Indian J. Exp. Biol. 2006, 44, $171-182$. [PubMed]

27. Shimada, T.; Inoue, K.; Suzuki, Y.; Kawai, T.; Azuma, E.; Nakajima, T.; Shindo, M.; Kurose, K.; Sugie, A.; Yamagishi, Y.; et al. Arylhydrocarbon receptor-dependent induction of liver and lung cytochromes P450 1A1, $1 \mathrm{~A} 2$, and $1 \mathrm{~B} 1$ by polycyclic aromatic hydrocarbons and polychlorinated biphenyls in genetically engineered C57BL/6J mice. Carcinogenesis 2002, 23, 1199-1207. [CrossRef] [PubMed]

28. You, L. Steroid hormone biotransformation and xenobiotic induction of hepatic steroid metabolizing enzymes. Chem. Biol. Interact. 2004, 147, 233-246. [CrossRef] [PubMed]

29. Ekuase, E.J.; Liu, Y.; Lehmler, H.J.; Robertson, L.W.; Duffel, M.W. Structure-activity relationships for hydroxylated polychlorinated biphenyls as inhibitors of the sulfation of dehydroepiandrosterone catalyzed by human hydroxysteroid sulfotransferase SULT2A1. Chem. Res. Toxicol. 2011, 24, 1720-1728. [CrossRef] [PubMed]

30. Ekuase, E.J.; Lehmler, H.J.; Robertson, L.W.; Duffel, M.W. Binding interactions of hydroxylated polychlorinated biphenyls (OHPCBs) with human hydroxysteroid sulfotransferase hSULT2A1. Chem. Biol. Interact. 2014, 212, 56-64. [CrossRef] [PubMed]

31. Liu, Y.; Apak, T.I.; Lehmler, H.J.; Robertson, L.W.; Duffel, M.W. Hydroxylated polychlorinated biphenyls are substrates and inhibitors of human hydroxysteroid sulfotransferase SULT2A1. Chem. Res. Toxicol. 2006, 19, 1420-1425. [CrossRef] [PubMed]

32. Qin, X.; Lehmler, H.J.; Teesch, L.M.; Robertson, L.W.; Duffel, M.W. Chlorinated biphenyl quinones and phenyl-2,5-benzoquinone differentially modify the catalytic activity of human hydroxysteroid sulfotransferase hSULT2A1. Chem. Res. Toxicol. 2013, 26, 1474-1485. [CrossRef] [PubMed]

33. Van den Hurk, P.; Kubiczak, G.A.; Lehmler, H.J.; James, M.O. Hydroxylated polychlorinated biphenyls as inhibitors of the sulfation and glucuronidation of 3-hydroxy-benzo[a]pyrene. Environ. Health Perspect. 2002, 110, 343-348. [CrossRef] [PubMed]

34. Soldin, O.P.; Makambi, K.H.; Soldin, S.J.; O'Mara, D.M. Steroid hormone levels associated with passive and active smoking. Steroids 2011, 76, 653-659. [CrossRef] [PubMed]

35. Tweed, J.O.; Hsia, S.H.; Lutfy, K.; Friedman, T.C. The endocrine effects of nicotine and cigarette smoke. Trends Endocrinol. Metab. 2012, 23, 334-342. [CrossRef] [PubMed]

36. Villard, P.H.; Herber, R.; Sérée, E.M.; Attolini, L.; Magdalou, J.; Lacarelle, B. Effect of cigarette smoke on UDP-glucuronosyltransferase activity and cytochrome P450 content in liver, lung and kidney microsomes in mice. Pharmacol. Toxicol. 1998, 82, 74-79. [CrossRef] [PubMed]

(C) 2016 by the authors; licensee MDPI, Basel, Switzerland. This article is an open access article distributed under the terms and conditions of the Creative Commons by Attribution (CC-BY) license (http://creativecommons.org/licenses/by/4.0/). 